\title{
Negative index and mode coupling in all-dielectric metamaterials at terahertz frequencies
}

\author{
Eric Akmansoy ${ }^{1,2,{ }^{*}}$ and Simon Marcellin ${ }^{1,2}$ \\ ${ }^{1}$ Institut d'Electronique Fondamentale, Univ. Paris-Sud, Universite Paris-Saclay, 91405 Orsay, France \\ ${ }^{2}$ UMR 8622, CNRS, 91405 Orsay, France
}

Received: 27 March 2018 / Received in final form: 10 July 2018 / Accepted: 18 July 2018

\begin{abstract}
We elucidate the role of the mode coupling of the Mie resonances in all-dielectric metamaterials to ensure a negative effective index at terahertz frequencies. We perform a study as a function of the lattice period and of the frequency overlapping of the modes of resonance. We show that negative effective refractive index requires sufficiently strong mode coupling and that for even more strong mode coupling, the first two modes of Mie resonances are degenerate; the effective refractive index is then undetermined. We also show that it is possible to obtain near-zero, or even null, effective index with a judicious adjustment of the mode coupling. Further, we discuss the mode coupling effect with hybridization in metamaterials.
\end{abstract}

Keywords: All-dielectric metamaterials / Negative index / Mode coupling / Near-zero refractive index

\section{Introduction}

All-dielectric metamaterials (ADMs) are the promising "inflection" of metamaterials to go beyond their limits. $\mathrm{ADMs}$ are an alternative to metallic metamaterials. The advantages of ADMs come from their low losses and their simple geometry: they do not suffer from Ohmic losses and thus they may benefit from low energy dissipation, specially, ceramics of high dielectric permittivity and high quality factor $[1,2]$. In the microwave, their quality factor is greater than that of metallic metamaterials [3], and it is consequently also the case in terahertz $(\mathrm{THz})$ and optical frequencies. ADMs are partly inspired by the work of Richtmyer who developed the theory of dielectric resonators, which is based on the fact that "the dielectric has the effect of causing the electromagnetic field [. . .] to be confined to the cylinder itself and the immediately surrounding region of space" [4]. Taking the matter further, O'Brien and Pendry opened the way for ADMs by considering the periodic lattice of high permittivity resonators (HPRs), thus demonstrating artificial magnetism in the microwave $[2,5]$. ADMs rely on the first two modes of the Mie resonances of HPR. The first mode results in resonant effective permeability that can have negative values, while the second one results in resonant effective permittivity that can also have negative values. When both are simultaneously negative, the ADM is called "double negative" (DNG) and its effective refractive index is then

\footnotetext{
* e-mail: eric.akmansoy@u-psud.fr
}

negative [6-11]. The unit cell of ADM thus comprises two subwavelength building blocks of simple geometry $[12,13]$. In analogy with chemical molecules, the unit cell is generally called a meta-dimer [14], and the two building blocks are called meta-atoms. As the two are different, the unit cell is a hetero-dimer.

The large applications of ADMs (for a review, see Ref. [15]) include perfect reflectors [16], perfect absorbers [17,18], zero-index metamaterials [19], optical magnetic mirror [20], and Fano resonances [21,22]. ADMs have been demonstrated from the microwave to the optical domain. Artificial magnetism [3,23-25] and negative effective refractive index $[26,27]$ have been, theoretically and experi-mentally, shown in the $\mathrm{GHz}$ regime. Even though artificial magnetism provided by ADMs has been experimentally demonstrated in the THz [28] and infrared ranges [29-32], DNG refractive index has not been yet demonstrated, which impedes ADMs to be the equivalent of their metallic counterpart. Besides, $\mathrm{THz}$ radiation is widely defined as electromagnetic radiation in the frequency range $0.3-3 \mathrm{THz}$. It permits the obtention of physical data that are not accessible using X-ray or infrared radiation and it thus finds many applications in imaging, security, quality inspection, chemical sensing, astronomy, etc. On their part, metamaterials have evolved towards the implementation of photonic components [33]. HPRs are well suited for metamaterial applications in the low $\mathrm{THz}$ frequency range [34].

In the following, we report on the mode coupling effect which plays a dominant role in the electromagnetic properties of metamaterials [35-39], notably, in the achievement of a negative effective index. Magnetic and 


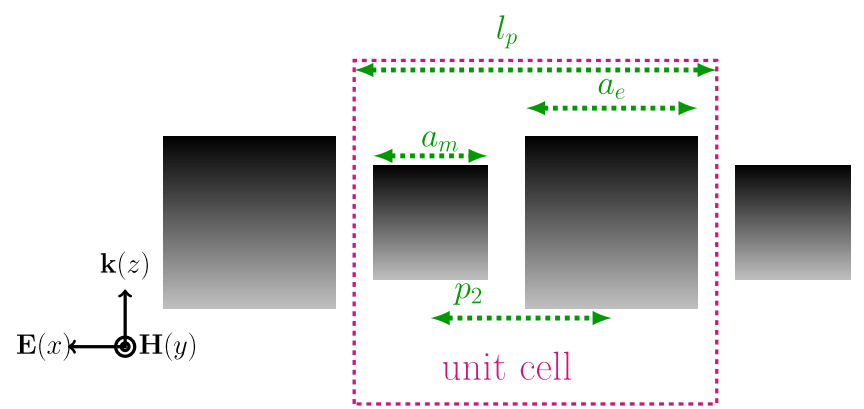

Fig. 1. Schematic layout of the 2D ADM (cross-sectional view). The ADM consists of one infinite layer along the $x$ direction and is made up of two interleaved sets of high permittivity square crosssection dielectric cylinders, which resonate in the first two modes of Mie resonances: the small set resonates in the magnetic mode and the second one in the electric mode. The equidistant cylinders are infinite along the $y$ direction and their side lengths are $a_{m}=60 \mu \mathrm{m}$ and $a_{e}=90 \mu \mathrm{m}$, respectively. Their relative permittivity is $\epsilon_{r}=94$. The unit cell actually consists of two subwavelength distinct building blocks. The lattice period is $l_{p}=260 \mu \mathrm{m}$ and $p_{2}$ is half the lattice period. The incident wave is transverse electric (TE).

electric mode coupling effects in ADMs have been separately studied in the microwave by Zhang et al. [36]. Herein, we report on the magneto-electric mode coupling effects in the $\mathrm{THz}$ domain, and we show that negative effective refractive index requires sufficiently strong mode coupling [40]. We also show that adjusting the mode coupling allows us to attain near-zero values of the refractive index, or even null effective index. Moreover, we highlight that the strongest values of the mode coupling lead to frequency mode degeneracy, for which the refractive index is undetermined.

\section{Simulations}

We consider a 2D ADM whose unit cell consists of two distinct building blocks, a magnetic block and an electric one, the former resonating in the first mode of Mie resonances and the latter resonating in the second mode $[3,13,27,41]$. The first mode is thus referred to as the magnetic mode and the second one as the electric mode. The ADM consists of one infinite layer made up of two sets of high permittivity square cross-section dielectric cylinders that are perpendicular to the incident wave vector (Fig. 1). The two sets of infinitely long HPRs are actually interleaved. The incident polarization is transverse electric (TE), i.e., the electric field is perpendicular to the axis of the cylinders. We studiy both spatial mode coupling and frequency mode coupling. The ADM has been numerically simulated by the means of the finite elements method software HFSS ${ }^{\mathrm{TM}}$, which yields the $S$ parameters. The side lengths of the resonators are initially $a_{m}=60 \mu \mathrm{m}$ for the magnetic mode and $a_{e}=$ $90 \mu \mathrm{m}$ for the electric one, while the lattice period is $l_{p}=260 \mu \mathrm{m}$. The HPRs are equidistant and therefore, the distance between two of them is half the lattice period $p_{2}=l_{p} / 2=130 \mu \mathrm{m}$. The relative permittivity of the dielectric is $\epsilon_{r}=94$ (titanium dioxide $-\mathrm{TiO}_{2}$ ) and the loss tangent increases between $\tan \delta=0.009$ and 0.015 in the considered frequency range $[42,43]$. We are thus dealing with a high refractive index bulk material $\left(N_{\mathrm{TiO}_{2}} \simeq 10\right)$.

\section{Results and discussion}

\subsection{Negative index and mode coupling}

We study the mode coupling between the first two modes of Mie resonances depending on the lattice period $l_{p}$ (spatial mode coupling), and then depending on the frequency overlapping of the two modes (frequency mode coupling). The results of the simulation, namely the minimum $n_{\text {eff }_{\text {min }}}$ of the real part of the effective index $n_{\text {eff }}$ as a function of the lattice period and as a function of the frequency spacing between the two modes, are reported in Figures 2 and 3, respectively. They show that the mode coupling should be strong enough to ensure negative effective index. The minima of the real part of the effective index $n_{\text {eff }}$ are $n_{\text {eff }_{\min }}=-2.2$ and -1.9 , respectively. On the one hand, increasing the mode coupling is obtained by decreasing the lattice period $l_{p}$. On the other hand, it is obtained by decreasing the frequency of the second mode of Mie resonances, which stems from the increasing of the side length $a_{e}$ of the electric resonator. When the mode coupling is sufficient, the bandwidth of the negative effective index $n_{\text {eff }}$ increases with it (see insets in Figs. 2 and 3). The frequency range of negative effective index is given by the relation [44]

$$
\epsilon^{\prime}(\omega) \cdot \mu^{\prime \prime}(\omega)+\epsilon^{\prime \prime}(\omega) \cdot \mu^{\prime}(\omega) \leq 0,
$$

where ' and " respectively denote the real and imaginary parts of the permeability $\mu$ and the permittivity $\epsilon$.

\subsection{Monomode coupling}

To carry out our study, we first explored monomode coupling, that is, the mode coupling due to only one mode, which arises inside a layer whose unit cell only consists of one building block, the magnetic one or the electric one. We study both cases. Consequently, we only consider the spatial mode coupling and only varied the lattice period $l_{p}$ which is equal to the distance $p_{1}$ between two resonators, $l_{p}=p_{1}$. Operating in the same frequency range, the side length of the magnetic resonator is $a_{m}=60 \mu \mathrm{m}$, while that of the electric resonator is $a_{e}=90 \mu \mathrm{m}$. The results of the simulation are reported in Figures 4 and 5, for both cases, and they show that the two modes behave differently. Their respective frequencies $\left(f_{m r}, f_{e r}\right)$ are given by the minima of the $S_{12}$ parameter [36]. The frequency $f_{m r}$ of the magnetic mode increases with the lattice period $l_{p}$, whereas the frequency $f_{e r}$ of the electric one decreases. These results are consistent with that of Zhang et al [36]. Both maxima of the absorption $A_{m r}$ and $A_{e r}\left(A(\omega)=1-\left|S_{12}(\omega)\right|^{2}-\right.$ $\left|S_{11}(\omega)\right|^{2}$ ) and the frequency of the resonance modes of the individual resonator are also reported. The latter provides a series of resonances whose frequency is 


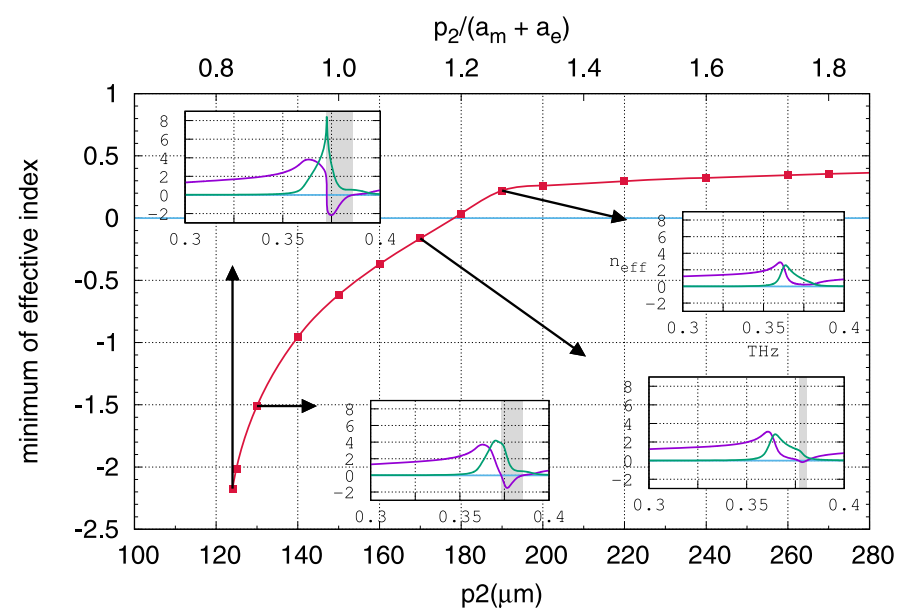

Fig. 2. Spatial mode coupling: minimal value of the effective refractive index $n_{\text {eff }}$ as a function of the distance $p_{2}$ between the two resonators. The side lengths of the resonators are $a_{m}=60 \mu \mathrm{m}$ and $a_{e}=90 \mu \mathrm{m}$, respectively. Insets: real (purple) and imaginary part (green) of the effective index $n_{\text {eff }}$; the shaded area denotes the bandwidth of negative effective index.

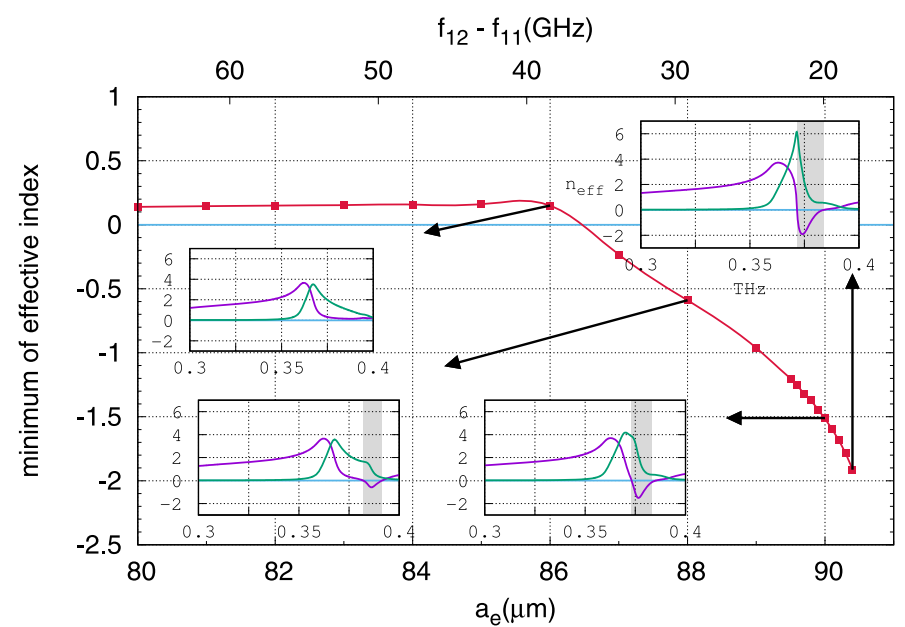

Fig. 3. Frequency mode coupling: minimal value of the effective refractive index $n_{\text {eff }}$ as a function of the side length $a_{e}$ of the electric resonator, namely, the frequency of the electric mode is varying. Insets: real (purple) and imaginary part of (green) the effective index $n_{\text {eff }}$; the shaded area denotes the bandwidth of negative effective index. $f_{11}$ and $f_{12}$ are the resonances frequencies of the individual resonators (see the text).

determined by Cohn's model $[1,26,45,46]$.

$$
f_{m n}=\frac{c}{2 \sqrt{\epsilon_{r}}} \sqrt{\left(\frac{m}{a}\right)^{2}+\left(\frac{n}{b}\right)^{2}},
$$

where $\epsilon_{r}$ is the relative permittivity of the resonator, $m$ and $n$ the integers, $a$ and $b$ the side lengths of the resonator, and $c$ is the velocity of light and its accuracy is about $5 \%$. Equation 2 was used to design all the reported structures. For a square cross-section cylinder, $a=b$, and the frequencies of the first two modes of the individual resonator respectively correspond to $m=n=1$ and $m=1$

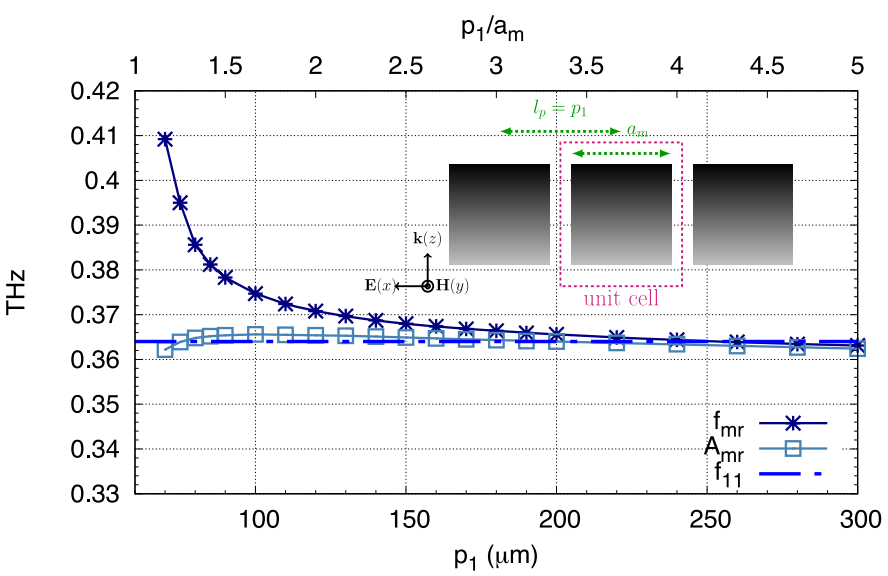

Fig. 4. Magnetic mode coupling: frequency $\left(f_{\mathrm{mr}}\right)$ of the first mode of Mie resonances and of the maximum of absorption $\left(A_{\mathrm{mr}}\right)$ as a function of the distance $p_{1}$ between two resonators. The unit cell only comprises the magnetic block. The side length of the resonator is $a_{m}=60 \mu \mathrm{m}$. The dashed line denotes the resonance frequency $\left(f_{11}\right)$ of the mode of resonances of the individual resonator.

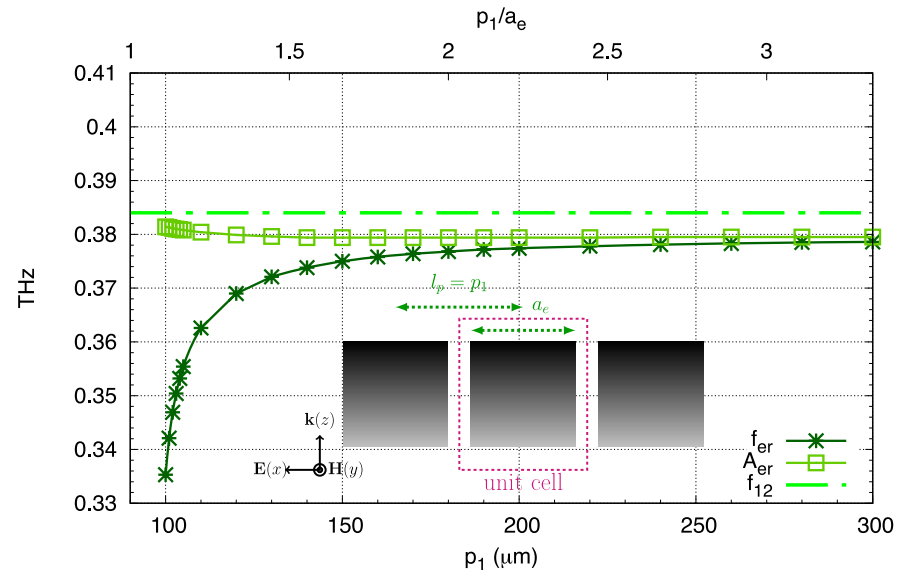

Fig. 5. Electric mode coupling: frequency $\left(f_{\mathrm{er}}\right)$ of the second mode of Mie resonances and of the maximum of absorption $\left(A_{\mathrm{er}}\right)$ as a function of the distance $p_{1}$ between two resonators. The unit cell only comprises the electric block. The side length of the resonator is $a_{e}=90 \mu \mathrm{m}$. The dashed line denotes the resonance frequency $\left(f_{12}\right)$ of the mode of resonances of the individual resonator.

and $n=2$. To exhibit negative refractive index, the involved resonance modes are the first mode of the magnetic resonator and the second mode of the electric resonator. Their frequencies are respectively $f_{11}=0.364$ $\mathrm{THz} \quad\left(a=a_{m}=60 \mu \mathrm{m}\right)$ and $f_{12}=0.384 \mathrm{THz} \quad\left(a=a_{e}=\right.$ $90 \mu \mathrm{m})[27]$. These are obviously constant, while the maxima of the absorption are nearly constant. It can be noticed that, as the mode coupling increases, the distance between the resonance frequencies $\left(f_{m r}, f_{e r}\right)$ of the resonator inside the layer and that $\left(f_{11}, f_{12}\right)$ of the individual resonator respectively increases (cf. Figs. 4 and 5, respectively), thus demonstrating the mode coupling. 


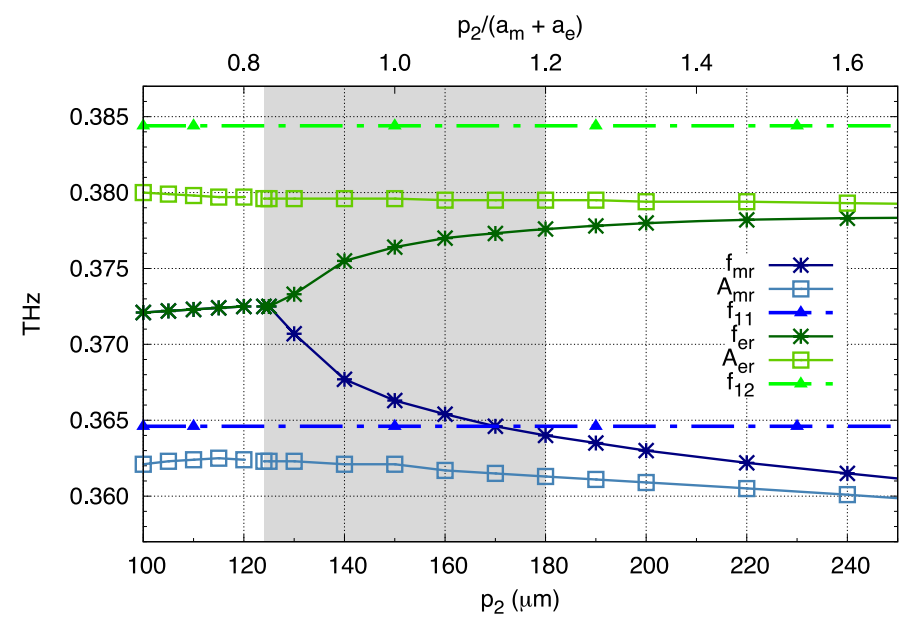

Fig. 6. Spatial mode coupling: frequency of the first two modes of Mie resonances as a function of the distance $p_{2}$ between two resonators which is half the lattice period $l_{p}$. Blue and green colors denote the magnetic and the electric modes, respectively. Square dots and crossed dots denote the maxima of absorption $\left(A_{\mathrm{mr}}, A_{\mathrm{er}}\right)$ and the minimum of the $S_{12}$ parameter $\left(f_{\mathrm{mr}}, f_{\mathrm{er}}\right)$, respectively. The shaded area corresponds to negative value of the effective index $n_{\text {eff. }}$ The side lengths of both resonators are $a_{m}=60 \mu \mathrm{m}$ and $a_{e}=90 \mu \mathrm{m}$, respectively. The dashed lines denote the frequencies of resonances $\left(f_{11}, f_{12}\right)$ of the modes of the individual resonator.

\subsection{Bimodal coupling}

We next explore the mode coupling inside the ADM, namely, the unit cell now consists of the two building blocks. The variation of the resonance frequencies $\left(f_{m r}, f_{e r}\right)$ of both modes is reported in Figures 6 and 7 corresponding to the spatial mode coupling and the frequency mode coupling, respectively. These frequencies are still given by the minima of the $S_{12}$ parameter [36]. Decreasing the lattice period $l_{p}$, i.e., the distance $p_{2}$ between the resonators, increases the mode coupling. Varying the overlapping of the two modes stems from the decreasing of the frequency of the electric mode, which also increases the mode coupling [35]. The curves are shaped as "tuning forks" and show that the frequencies $\left(f_{m r}, f_{e r}\right)$ of the two modes of resonance are moving closer together as the mode coupling increases. To highlight this effect, the frequencies $\left(f_{11}, f_{12}\right)$ of the resonance modes of the individual resonator are again shown in these figures. For both the magnetic mode and the electric one, the distance between the frequency $\left(f_{m r}, f_{e r}\right)$ of the resonator inside the $\mathrm{ADM}$ and the frequency $\left(f_{11}, f_{12}\right)$ of the individual one respectively increases as the mode coupling increases. This anew evidences the mode coupling inside the ADM. Theses curves also show that further increasing the mode coupling gives rise to a frequency degeneracy in both cases of mode coupling, that is, the two resonance frequencies of the two modes become equal, $f_{\mathrm{mr}}=f_{\mathrm{er}}$.

The $S_{12}$ parameter and the absorption $A$ are reported in Figure 8 as a function of frequency for several values of lattice period $l_{p}$, which is relative to the spatial mode coupling. Two ranges of lattice period $l_{p}$ are considered, one is out of the frequency degeneracy regime $\left(250 \leq l_{p} \leq\right.$

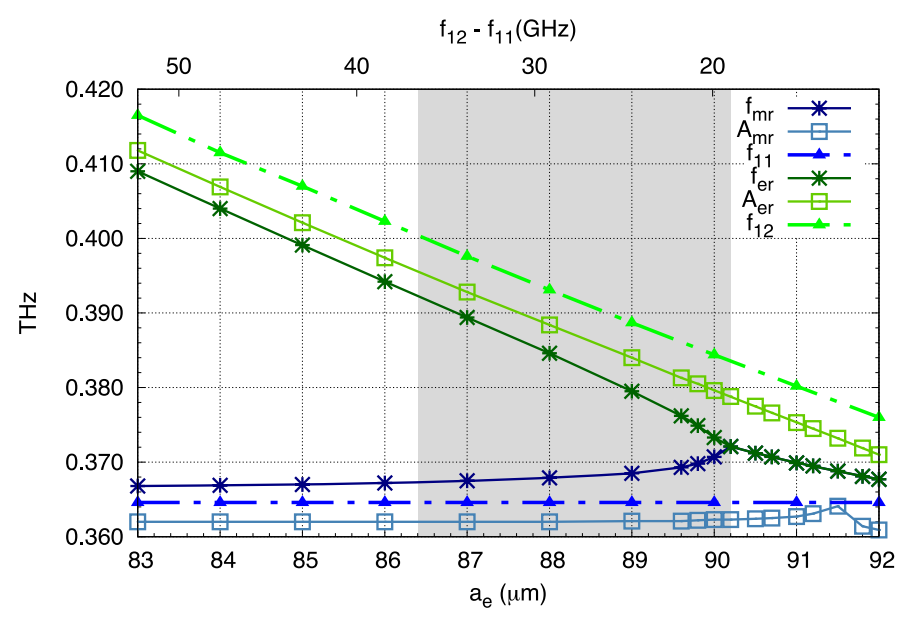

Fig. 7. Frequency mode coupling: frequency of the first two modes of Mie resonances $\left(f_{\mathrm{mr}}, f_{\mathrm{er}}\right)$ as a function of the side length $a_{e}$ of the electric resonator, namely, the frequency of the electric mode is varying. The convention is the same as in Figure 6. The side length of the magnetic resonator is $a_{m}=60 \mu \mathrm{m}$ and the lattice period $l_{p}$ is $260 \mu \mathrm{m}$.

$400 \mu \mathrm{m})$ and the second one in the frequency degeneracy regime $\left(200 \leq l_{p} \leq 248 \mu \mathrm{m}\right)$. It can be noticed that the frequency of both maxima of absorption are practically constant, whereas the frequency of both minima of the $S_{12}$ parameter vary with the lattice period $l_{p}$. The latter move closer together as the lattice period $l_{p}$ increases, until they merged, which corresponds to the frequency degeneracy. The crossing point is reached when the lattice period is equal to $l_{p c}=250 \mu \mathrm{m}$. The merged minima of the $S_{12}$ parameter are very weak $(\lesssim-51 \mathrm{~dB})$ for $l_{p}=248 \mu \mathrm{m}$, which corresponds to the minimum of the real part of the effective index $n_{\mathrm{eff}_{\min }}\left(l_{p}=248 \mu \mathrm{m}\right)=-2.2$. (See effective index curves inserted in Figs. 2 and 3 which are continuous, but at the limit of continuity.) For greater values of the mode coupling, that is, for lattice period $l_{p}$ smaller than $248 \mu \mathrm{m}$, the effective parameters could not be extracted, being not continuous through all frequencies [47]; the effective refractive index $n_{\text {eff }}$ is then undetermined. We observed the same frequency degeneracy behavior when studying the frequency mode coupling (results not shown).

The mode coupling effect we report here is different from hybridization [48] which is observed with plasmonic metameterial [14,49], split-ring resonators metametarials [50], inductor-capacitor resonators [51], cut wires [52], nano-wires [53], nano-rings [54], nano-particle dimers [55], or silicon nanoparticles [56]. In these cases, the coupling between the two identical meta-atoms which constitute the dimer leads from a trapped mode to the formation of new hybridized modes because it lifts the degeneracy of the mode of the individual meta-atoms. Hybridization may be used to yield negative refractive index [50,51,54]. The unit cell is then a homo-dimer and the negative effective index is achieved by playing with the mode coupling so as to overlap two hybridized modes which are of different kind: magnetic orelectric. In the case we report here, the mechanism is different since it takes the reverse way, 


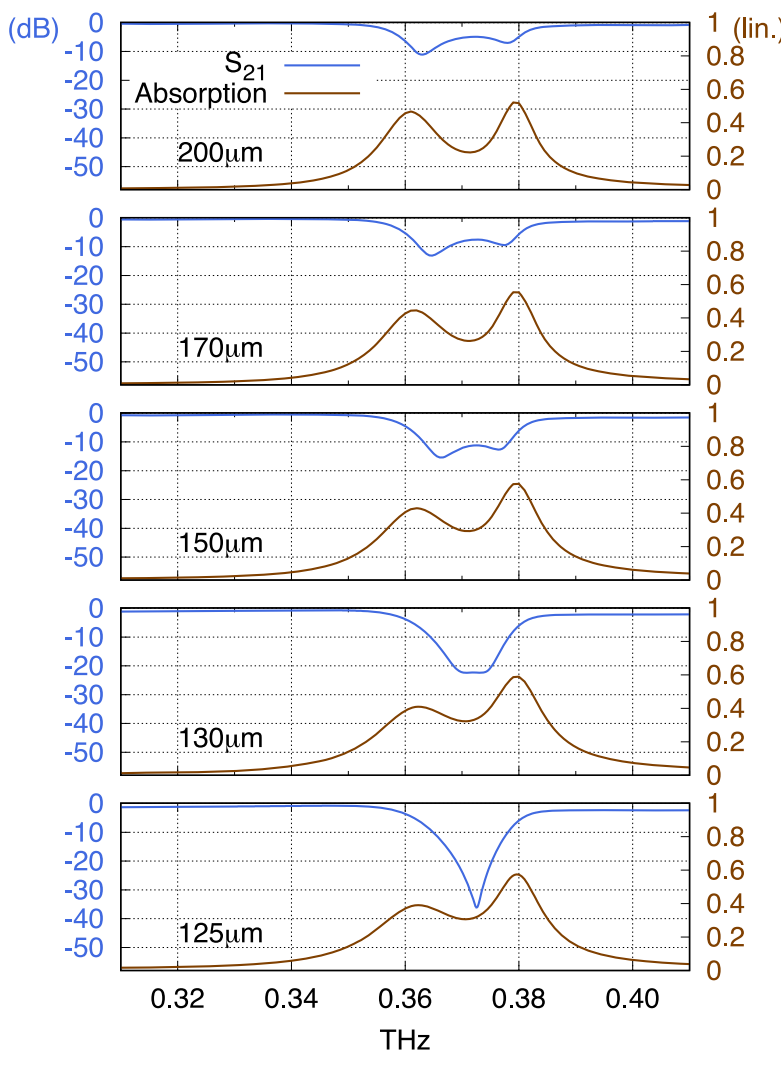

(a)

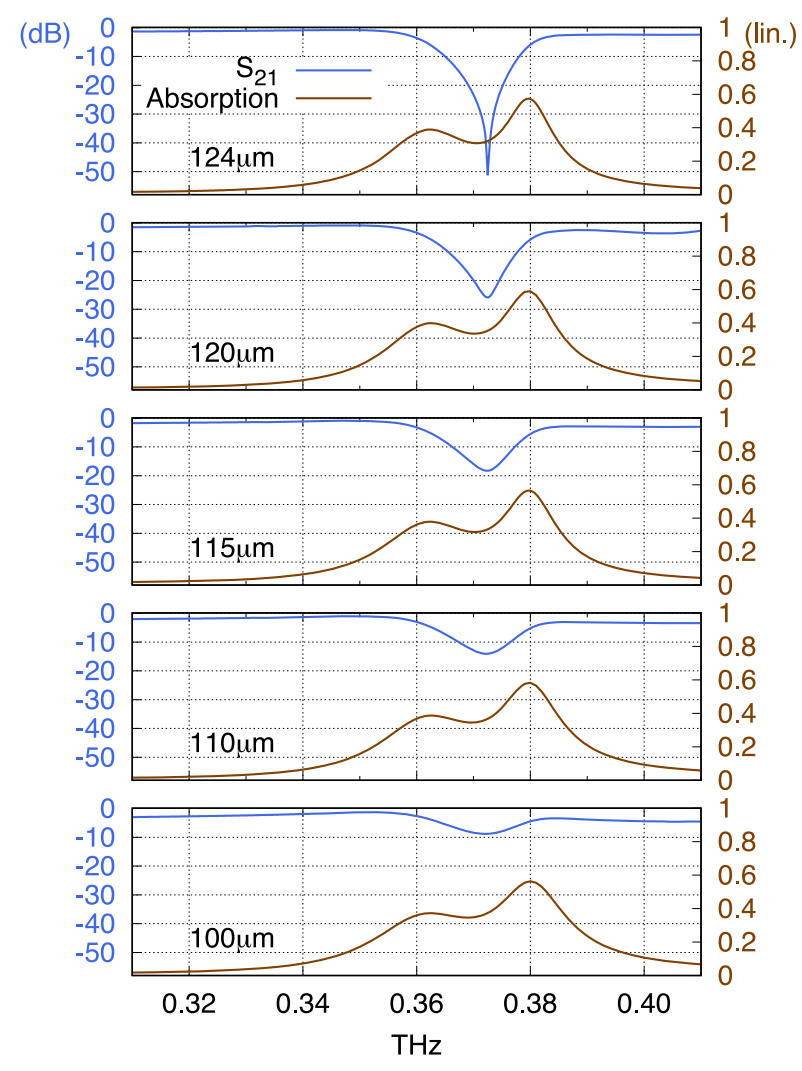

(b)

Fig. 8. Effect of spatial mode coupling: $S_{12}$ parameter (log. scale (dB)) and absorption $A$ (linear scale (lin.)) for several values of distance $p_{2}$ between two resonators. The side lengths of the resonators are $a_{m}=60 \mu \mathrm{m}$ and $a_{e}=90 \mu \mathrm{m}$. (a) Out of frequency degeneracy regime $\left(125 \leq p_{2} \leq 200 \mu \mathrm{m}\right)$; (b) in frequency degeneracy regime $\left(100 \leq p_{2} \leq 124 \mu \mathrm{m}\right)$. The merged minima of the $S_{12}$ parameter attain very weak values $(\lesssim-51 \mathrm{~dB})$ when $p_{2}=p_{2 c}=124 \mu \mathrm{m}$, which correspond to the minimum of the refractive index $n_{\text {eff }_{\min }}=-2.2$.

because increasing the mode coupling leads from two separate modes to a trapped one. The unit cell of our ADM is a hetero-dimer because the two meta-atoms are not identical, and moreover, each one resonates in a different kind of mode: the magnetic one and the electric one. Hence, increasing the mode coupling leads to the trapped mode putting together the two separate modes. Nevertheless, the mode coupling has to be strong enough to ensure a negative effective index, the unit cell being either a homo-dimer or a hetero-dimer.

\subsection{Effective parameters}

In our ADM, a magnetic moment ensues from the magnetic mode giving rise to resonant effective permeability $\mu_{\text {eff }}$. Similarly, an electric moment ensues from the electric mode giving rise to resonant effective permittivity $\epsilon_{\text {eff }}$. The two are perpendicular to each other (see e.g., Fig. 1 in reference [41]). The mode coupling arises from the interaction between these electromagnetic moments and it changes with the separating distance. The side lengths of the resonators are afresh $a_{m}=60 \mu \mathrm{m}$ and $a_{e}=90 \mu \mathrm{m}$. Both resonances consequently modify the effective refractive index $n_{\text {eff }}(\omega)$ of the ADM. The effective electromagnetic parameters $\mu_{\text {eff }}, \epsilon_{\text {eff }}$ and $n_{\text {eff }}$ are reported in Figure 9 relative to two values of the lattice period $l_{p}=360 \mu \mathrm{m}$ and $l_{p}=260 \mu \mathrm{m}$. They are extracted from the $S$-parameters using the common retrieval method described in references [47,57-63]. The antiresonance behavior of the effective permittivity $e_{\text {eff }}$ around the magnetic mode frequency, which is inherent in metamaterials, can be observed [62-65]. In the former case (low mode coupling), the real part of the effective index $n_{\text {eff }}$ does not reach negative values, whereas it does in the latter case (strong mode coupling), then satisfying equation 1 . The minimum value of the real part of the effective index is then $n_{\text {eff }_{\text {min }}}\left(l_{p}=260 \mu \mathrm{m}\right)=-1.5$. In the former case, the real part of the effective index $n_{\text {eff }}$ is below unity and close to zero. Its minimal value is $n_{\text {eff }_{\min }}\left(l_{p}=360 \mu \mathrm{m}\right) \lesssim 0.04$, demonstrating that adjusting the mode coupling makes ADMs suitable for epsilon-near-zero (ENZ) metamaterials $[66,67]$ (see also Fig. 2), or even null effective index [19]. It can also be noticed that the mode coupling strongly enhances the amplitude of both resonances, notably the electric one, and that it brings closer together the two modes. In addition, as we are dealing with a high refractive index bulk material $\left(N_{\mathrm{TiO}_{2}} \simeq 10\right)$, the wavelength inside it is about one-tenth of that in vacuum. Consequently, we calculated the static effective permittivity, i.e., beyond the resonances, from the Maxwell-Garnett model [68]. It is equal to $\epsilon_{\text {eff }}=2.1$ and $\epsilon_{\text {eff }}=2.9$ corresponding to both values of the lattice period 


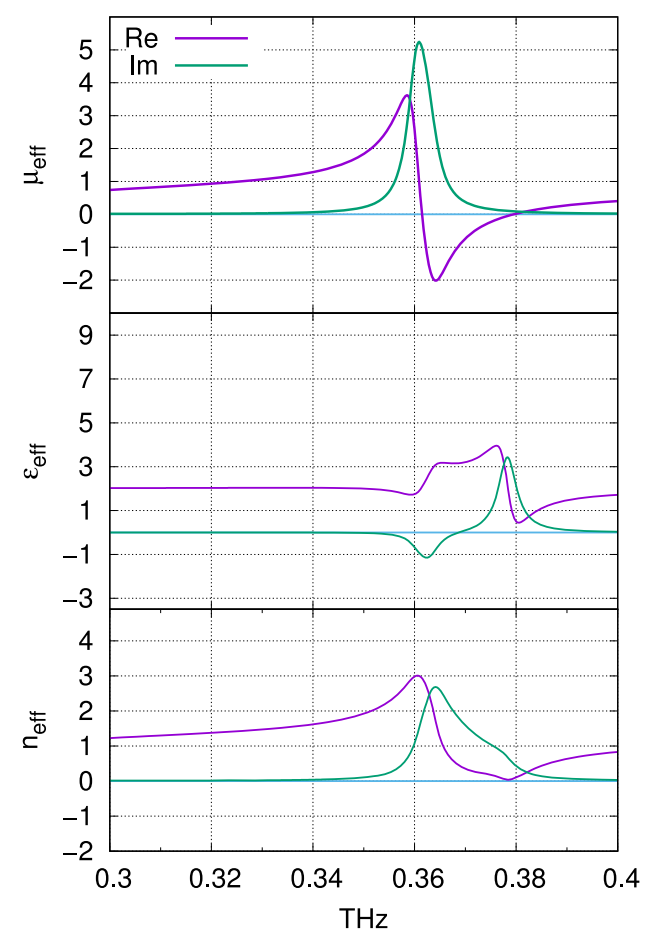

(a)

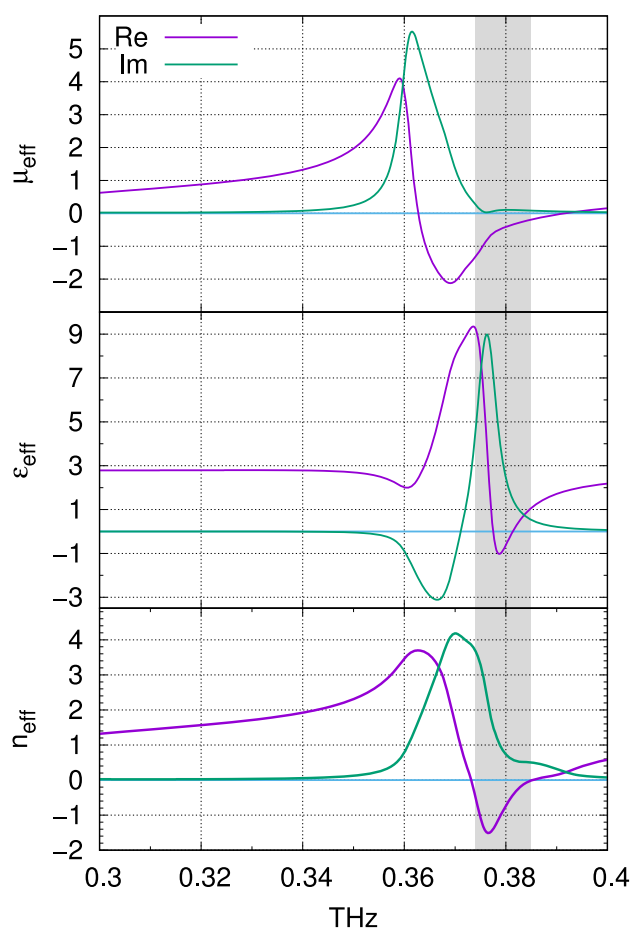

(b)

Fig. 9. Effect of spatial mode coupling: effective electromagnetic parameters (real and imaginary parts): permeability $\mu_{\text {eff }}$ (top), permittivity $\epsilon_{\text {eff }}$ (middle) and effective index $n_{\text {eff }}$ (bottom). (a) Low mode coupling: lattice period $l_{p}=360 \mu \mathrm{m}$ : the minimum value of the effective refractive index is $n_{\text {eff }_{\min }}<0.04$; (b) strong mode coupling: lattice period $l_{p}=260 \mu \mathrm{m}$. The shaded area denotes the bandwidth of the negative effective index: the minimum value of the effective refractive index is $n_{\text {eff }_{\text {min }}}=-1.5$. The static effective permittivity values yielded by the Maxwell-Garnett formula, $\epsilon_{\text {eff }}=2.1$ and $\epsilon_{\text {eff }}=2.9$, are in good agreement with the result of the simulation.

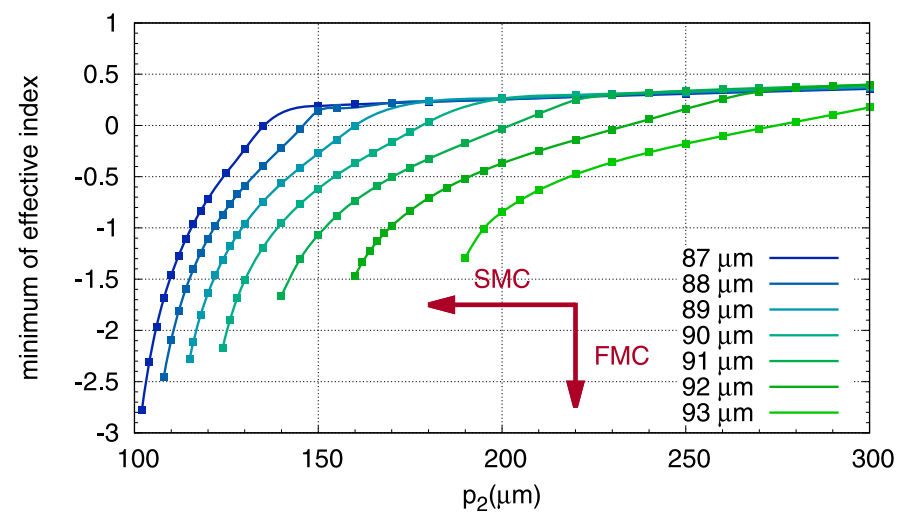

Fig. 10. Effect of both mode couplings (spatial mode coupling (SMC) and frequency mode coupling (FMC)): minimal value of the effective refractive index $n_{\mathrm{eff}}$ as a function of the distance $p_{2}$ between two resonators for several values of the side length $a_{e}$ of the electric resonator. The side length of the magnetic resonator is $a_{m}=60 \mu \mathrm{m}$. The two arrows denote increasing mode coupling. The minimum of the real part of the effective index is $n_{\text {eff }_{\min }}=-2.8$.

$l_{p}=360 \mu \mathrm{m}$ and $l_{p}=260 \mu \mathrm{m}$, respectively, which is in good agreement with the results of the simulation (see Fig. 9a and b-middle).

To engineer the electromagnetic properties of an ADM, one can consequently play with either mode couplings: the spatial mode coupling or the frequency mode coupling. Figure 10 gathers the role of both mode couplings: the stronger the two mode couplings, the more negative the effective index and the larger the bandwidth of negative effective index (cf. Figs. 2 and 3). Combining the two mode couplings, negative effective index as low as $n_{\text {eff }}=-2.8$ is obtained.

\subsection{Dielectric function, phonons and strontium titanate $\left(\mathrm{SrTiO}_{3}\right)$}

Other high permittivity materials, having low losses, can be investigated to study the mode coupling inside ADMs at $\mathrm{THz}$ frequencies, e.g., $\mathrm{SrTiO}_{3}$ [69-72]. However, the dielectric function $\epsilon_{r}(\omega)$ of these high permittivity materials is dispersive, because of lattice vibrations, namely, due to optical phonons [73]. Their frequency is in the $\mathrm{THz}$ range, and we are concerned with the transverse optical phonon of lowest frequency $\left(\mathrm{TO}_{1}\right)$. The $\mathrm{TO}_{1}$ phonon frequency of $\mathrm{SrTiO}_{3}$ is $2.70 \mathrm{THz}$ [69], while that of $\mathrm{TiO}_{2}$ is $5.6 \mathrm{THz}[69,73]$. The dielectric function $\epsilon_{r}(\omega)$ is described by the classical oscillator model or the FourParameter Semi Quantum (FPSQ) model [69,71,73]. Measurements in the $\mathrm{THz}$ frequency, reported in the literature, are in good agreement with these models for $\mathrm{TiO}_{2}[42,43]$ and $\mathrm{SrTiO}_{3}[71,72,74,75]$. The two yield the same dielectric function $\epsilon_{r}(\omega)$ at the operating frequency. 
The latter also reflect that the imaginary part of the dielectric function $\epsilon_{r}(\omega)$ strongly increases around the frequency of the $\mathrm{TO}_{1}$ phonon. We also simulated a similar ADM but consisting of HPRs made of $\mathrm{SrTiO}_{3}$ and found that it exhibits the same mode coupling effects (results not shown here). The real part of the effective index reaches values as low as $n_{\text {eff }_{\text {min }}}=-7$, meaning that the effect of the mode coupling is stronger when a higher permittivity material is used ( $\epsilon_{r} \simeq 300$ [72] as compared with $\left.\epsilon_{r}=94\right)$, because this heightens the resonances.

However, losses (imaginary part of $\epsilon_{r}(\omega)$ ) resulting from the $\mathrm{TO}_{1}$ phonon greatly increase and therefore limit the operating range at $\mathrm{THz}$ frequencies. In addition, the real part of the dielectric function $\epsilon_{r}(\omega)$ falls down at higher frequency [72,74], which drastically modifies the Mie resonances. The lower permittivity of $\mathrm{TiO}_{2}$ leads to greater side lengths $a_{m}$ and $a_{e}$ of each resonator (see Eq. (2)) and therefore, it facilitates their fabrication. Consequently, $\mathrm{TiO}_{2}$ is more suitable for $\mathrm{ADM}$ applications at $\mathrm{THz}$ frequencies.

\section{Conclusion}

We have studied mode coupling effects in ADMs at $\mathrm{THz}$ frequencies, and we show that the mode coupling has to be sufficiently strong to ensure negative effective index of refraction. Tuning the first two modes of Mie resonances of an ADM by adjusting the mode coupling allows the setting of the effective index from a near-zero value to a negative value. We studied both spatial mode coupling and frequency mode coupling. Increasing both brings the modes closer together until they are merged. Thus, we highlight the frequency degeneracy of the first two resonance modes, namely, the two frequencies are equal, and the effective index is then undetermined. At the crossing point, the effective index reaches its lowest value.

\section{Author contribution statement}

E.A. supervised the study, analyzed the results and wrote the paper. S.M. made the simulation and analyzed the results.

The authors thank Aloyse Degiron for helpful discussion. E.A. thanks Fabrice Rossignol and Jean-Pierre Ganne for their help. This work has been funded by the Agence Nationale de la Recherche TeraMetaDiel project (number ANR-12-BS03-0009).

\section{References}

1. D. Kajfez, P. Guillon, Dielectric Resonators, (Artech House, Dedham, MA, 1986)

2. S. Fiedziuszko, I. Hunter, T. Itoh, Y. Kobayashi, T. Nishikawa, S. Stitzer, K. Wakino, Dielectric materials, devices, and circuits, IEEE Trans. Microw. Theory Tech. 50, 706 (2002)

3. B.-I. Popa, S.A. Cummer, Compact dielectric particles as a building block for low-loss magnetic metamaterials, Phys. Rev. Lett. 100, 207401 (2008)
4. R.D. Richtmyer, Dielectric resonators, J. Appl. Phys. 10, 391 (1939)

5. S. O'Brien, J.B. Pendry, Photonic band-gap effects and magnetic activity in dielectric composites, J. Phys.: Condens. Matter 14, 15 (2002)

6. J.B. Pendry, D.R. Smith, Reversing light with negative refraction, Phys. Today 57, 37 (2004)

7. W.J. Padilla, D.N. Basov, D.R. Smith, Negative refractive index metamaterials, Mater. Today 9, 28 (2006)

8. D.R. Smith, W.J. Padilla, D.C. Vier, S.C. Nemat-Nasser, S. Schultz, Composite medium with simultaneously negative permeability and permittivity, Phys. Rev. Lett. 84, 4184 (2000)

9. R.W. Ziolkowski, Design, fabrication, and testing of double negative metamaterials, IEEE Trans Antenn Propag. 51, 1516 (2003)

10. N. Engheta, R.W. Ziolkowski, A positive future for doublenegative metamaterials, IEEE Trans. Microw. Theory Tech. 53, 1535 (2005)

11. R. Liu, A. Degiron, J.J. Mock, D.R. Smith, Negative index material composed of electric and magnetic resonators, Appl. Phys. Lett. 90, 263504 (2007)

12. A. Ahmadi, H. Mosallaei, Physical configuration and performance modeling of all-dielectric metamaterials, Phys. Rev. B 77, 045104 (2008)

13. Q. Zhao, J. Zhou, F. Zhang, D. Lippens, Mie resonance-based dielectric metamaterials, Mater. Today 12, 60 (2009)

14. N. Liu, H. Liu, S. Zhu, H. Giessen, Stereometamate-rials, Nat. Photonics 3, 157 (2009)

15. S. Jahani, Z. Jacob, All-dielectric metamaterials, Nat Nano 11, 23 (2016)

16. P. Moitra, B.A. Slovick, W. Li, I.I. Kravchencko, D.P. Briggs, S. Krishnamurthy, J. Valentine, Large-scale all-dielectric metamaterial perfect reflectors, ACS Photonics 2, 692 (2015)

17. X. Liu, Q. Zhao, C. Lan, J. Zhou, Isotropic mie resonancebased metamaterial perfect absorber, Appl. Phys. Lett. 103, 031910 (2013)

18. X. Liu, K. Bi, B. Li, Q. Zhao, J. Zhou, Metamaterial perfect absorber based on artificial dielectric "atoms", Opt. Express 24, 20454 (2016)

19. P. Moitra, Y. Yang, Z. Anderson, I.I. Kravchenko, D.P. Briggs, J. Valentine, Realization of an all-dielectric zero-index optical metamaterial, Nat. Photon. 7, 791 (2013)

20. S. Liu, M.B. Sinclair, T.S. Mahony, Y.C. Jun, S. Cam-pione, J. Ginn, D.A. Bender, J.R. Wendt, J.F. Ihlefeld, P.G. Clem, J.B. Wright, I. Brener, Optical magnetic mirrors without metals, Optica 1, 250 (2014)

21. B. Luk'yanchuk, N.I. Zheludev, S.A. Maier, N.J. Ha-las, P. Nordlander, H. Giessen, C.T. Chong, The fano resonance in plasmonic nanostructures and metama-terials, Nat. Mater. 9, 707 (2010).

22. T. Lepetit, E. Akmansoy, J.-P. Ganne, J.-M. Lour-tioz, Resonance continuum coupling in high-permittivity dielectric metamaterials, Phys. Rev. B 82, 195307 (2010)

23. Q. Zhao, L. Kang, B. Du, H. Zhao, Q. Xie, X. Huang, B. Li, J. Zhou, L. Li, Experimental demonstration of isotropic negative permeability in a three-dimensional dielectric composite, Phys. Rev. Lett. 101, 027402 (2008)

24. T. Lepetit, E. Akmansoy, M. Pate, J.P. Ganne, Broadband negative magnetism from all-dielectric meta-material, Electron. Lett. 44, 1119 (2008)

25. I. Vendik, M. Odit, D. Kozlov, 3D isotropic metama-terial based on a regular array of resonant dielectric spherical inclusions, Metamaterials 3, 140 (2009) 
26. J. Kim, A. Gopinath, Simulation of a metamaterial containing cubic high dielectric resonators, Phys. Rev. B 76, 115126 (2007)

27. T. Lepetit, E. Akmansoy, J.-P. Ganne, Experimental measurement of negative index in an all-dielectric metamaterial, Appl. Phys. Lett. 95, 121101 (2009)

28. H. Nemec, P. Kuzel, F. Kadlec, C. Kadlec, R. Yahiaoui, P. Mounaix, Tunable terahertz metamaterials with negative permeability, Phys. Rev. B 79, 241108 (2009)

29. M.S. Wheeler, J.S. Aitchison, J.I.L. Chen, G.A. Ozin, M. Mojahedi, Infrared magnetic response in a random silicon carbide micropowder, Phys. Rev. B 79, 073103 (2009)

30. J.C. Ginn, I. Brener, D.W. Peters, J.R. Wendt, J.O. Stevens, P. F. Hines, L.I. Basilio, L.K. Warne, J.F. Ih-lefeld, P.G. Clem, M. B. Sinclair, Realizing optical magnetism from dielectric metamaterials, Phys. Rev. Lett. 108, 097402 (2012)

31. J.A. Schuller, R. Zia, T. Taubner, M.L. Brongersma, Dielectric metamaterials based on electric and magnetic resonances of silicon carbide particles, Phys. Rev. Lett. 99, 107401 (2007)

32. L. Shi, T.U. Tuzer, R. Fenollosa, F. Meseguer, A new dielectric metamaterial building block with a strong magnetic response in the sub-1.5-micrometer region: silicon colloid nanocavities, Adv. Mater. 24, 5934 (2012)

33. N.I. Zheludev, Y.S. Kivshar, From metamaterials to metadevices, Nat. Mater. 11, 917 (2012)

34. F. Gaufillet, S. Marcellin, E. Akmansoy, Dielectric metamaterial-based gradient index lens in the terahertz frequency range, IEEE J. Sel. Top. Quantum Electron. 23, 1 (2017)

35. N. Liu, H. Giessen, Coupling effects in optical metamaterials, Angew. Chem. In t. Ed. 49, 9838 (2010)

36. F. Zhang, L. Kang, Q. Zhao, J. Zhou, D. Lip-pens, Magnetic and electric coupling effects of dielectric metamaterial, New J. Phys. 14, 033031 (2012)

37. F. Zhang, V. Sadaune, L. Kang, Q. Zhao, J. Zhou, D. Lippens, Coupling effect for dielectric metamate-rial dimer, Prog. Electromagn. Res. 132, 587 (2012)

38. S. Lanneb'ere, S. Campione, A. Aradian, M. Albani, F. Capolino, Artificial magnetism at terahertz frequencies from three-dimensional lattices of $\mathrm{TiO}_{2}$ microspheres accounting for spatial dispersion and magnetoelectric coupling, J. Opt. Soc. Am. B 31, 1078 (2014)

39. P. Albella, M.A. Poyli, M.K. Schmidt, S.A. Maier, F. Moreno, J.J. Śaenz, J. Aizpurua, Low-loss electric and magnetic fieldenhanced spectroscopy with subwave-length silicon dimers, J. Phys. Chem. C 117, 13573 (2013)

40. S. Marcellin, E. Akmansoy, Negative index and mode coupling in all-dielectric metamaterials at terahertz frequencies, in 2015 9th International Congress on Advanced Electromagnetic Materials in Microwaves and Optics (Metamaterials), Oxford, September 2015, pp. 4-6.

41. T. Lepetit, E. Akmansoy, J.-P. Ganne, Experimental evidence of resonant effective permittivity in a dielectric metamaterial, J. Appl. Phys. 109, 023115 (2011)

42. N. Matsumoto, T. Hosokura, K. Kageyama, H. Takagi, Y. Sakabe, M. Hangyo, Analysis of dielectric response of $\mathrm{TiO}_{2}$ in terahertz frequency region by general harmonic oscillator model, Jpn. J. Appl. Phys. 47, 7725 (2008)

43. K. Berdel, J. Rivas, P. Bolivar, P. de Maagt, H. Kurz, Temperature dependence of the permittivity and loss tangent of high-permittivity materials at terahertz frequencies, IEEE Trans. Microw. Theory Tech. 53, 1266 (2005)
44. R.A. Depine, A. Lakhtakia, A new condition to identify isotropic dielectric-magnetic materials displaying negative phase velocity, Microw. Opt. Tech. Lett. 41, 315 (2004)

45. S.B. Cohn, Microwave bandpass filters containing high-q dielectric resonators, IEEE Trans. Microw. Theory Tech. 16, 218 (1968)

46. J. Sethares, S. Naumann, Design of microwave dielectric resonators, IEEE Trans. Microw. Theory Tech. MT14, 2 (1966)

47. X. Chen, T.M. Grzegorczyk, B.-I. Wu, J. Pacheco, J.A. Kong, Robust method to retrieve the constitutive effective parameters of metamaterials, Phys. Rev. E 70, 016608 (2004)

48. E. Prodan, C. Radloff, N.J. Halas, P. Nordlander, A hybridization model for the plasmon response of complex nanostructures, Science 302, 5644419 (2003)

49. H. Guo, N. Liu, L. Fu, T.P. Meyrath, T. Zentgraf, H. Schweizer, H. Giessen, Resonance hybridization in double split-ring resonator metamaterials, Opt. Express 15, 12095 (2007)

50. R. Abdeddaim, A. Ourir, J. de Rosny, Realizing a negative index metamaterial by controlling hybridization of trapped modes, Phys. Rev. B 83, 033101 (2011)

51. N.-H. Shen, L. Zhang, T. Koschny, B. Dastmalchi, M. Kafesaki, C.M. Soukoulis, Discontinuous design of negative index metamaterials based on mode hybridization, Appl. Phys. Lett. 101, 081913 (2012)

52. N. Liu, H. Guo, L. Fu, S. Kaiser, H. Schweizer, H. Giessen, Plasmon hybridization in stacked cut-wire metamaterials, Adv. Mater. 19, 3628 (2007)

53. A. Christ, O.J.F. Martin, Y. Ekinci, N.A. Gippius, S.G. Tikhodeev, Symmetry breaking in a plasmonic metamaterial at optical wavelength, Nano Lett. 8, 2171 (2008)

54. B. Kante, Y.-S. Park, K. O'Brien, D. Shuldman, N.D. Lanzillotti-Kimura, Z. Jing Wong, X. Yin, X. Zhang, Symmetry breaking and optical negative index of closed nanorings, Nat. Commun. 3, 1180 (2012)

55. P. Nordlander, C. Oubre, E. Prodan, K. Li, M.I. Stockman, Plasmon hybridization in nanoparticle dimers, Nano Lett. 4, 899 (2004)

56. J. van de Groep, T. Coenen, S.A. Mann, A. Polman, Direct imaging of hybridized eigenmodes in coupled silicon nanoparticles, Optica 3, 93 (2016)

57. A.M. Nicolson, G.F. Ross, Measurement of the intrinsic properties of materials by time-domain techniques, IEEE Trans. Instrum. Meas. 19, 377 (1970)

58. W. Weir, Automatic measurement of complex dielectric constant and permeability at microwave frequencies, Proc. IEEE 62, 33 (1974)

59. Z. Szabo, G.H. Park, R. Hedge, E.P. Li, A unique extraction of metamaterial parameters based on Kramers-Kronig relationship, IEEE Trans. Microw. Theory Tech. 58, 2646 (2010)

60. V. Varadan, R. Ro, Unique retrieval of complex permittivity and permeability of dispersive materials from reflection and transmitted fields by enforcing causality, IEEE Trans. Microw. Theory Tech. 55, 2224 (2007)

61. D.R. Smith, S. Schultz, P. Markǒs, C.M. Soukoulis, Determination of effective permittivity and permeability of metamaterials from reflection and transmission coefficients, Phys. Rev. B 65, 195104 (2002)

62. T. Koschny, P. Markǒs, D.R. Smith, C.M. Souk-oulis, Resonant and antiresonant frequency dependence of the effective parameters of metamaterials, Phys. Rev. E 68, 065602 (2003) 
63. D.R. Smith, D.C. Vier, T. Koschny, C.M. Souk-oulis, Electromagnetic parameter retrieval from inhomo-geneous metamaterials, Phys. Rev. E 71, 036617 (2005)

64. A. Alú, Restoring the physical meaning of metamaterial constitutive parameters, Phys. Rev. B 83, 081102 (2011)

65. C.R. Simovski, On electromagnetic characterization and homogenization of nanostructured metamaterials, J. Opt. 13, 013001 (2011)

66. R.W. Ziolkowski, Propagation in and scattering from a matched metamaterial having a zero index of refraction, Phys. Rev. E 70, 046608 (2004)

67. A. Alú, M.G. Silveirinha, A. Salandrino, N. En-gheta, Epsilon-near-zero metamaterials and electromagnetic sources: tailoring the radiation phase pattern, Phys. Rev. B 75, 155410 (2007)

68. A. Sihvola, Metamaterials and depolarization factors, Prog. Electromagn. Res. 51, pp. 65-82, (2005)

69. W.G. Spitzer, R.C. Miller, D.A. Kleinman, L.E. Howarth, Far infrared dielectric dispersion in $\mathrm{BaTiO}_{3}, \mathrm{SrTiO}_{3}$, and $\mathrm{TiO}_{2}$, Phys. Rev. 126, 1710 (1962)

70. A.S. Barker, Temperature dependence of the transverse and longitudinal optic mode frequencies and charges in $\mathrm{srtio}_{3}$ and $\mathrm{BaTiO}_{3}$, Phys. Rev. 145, 391 (1966)
71. J. Han, F. Wan, Z. Zhu, W. Zhang, Dielectric response of soft mode in ferroelectric $\mathrm{SrTiO}_{3}$, Appl. Phys. Lett. 90, 031104 (2007)

72. N. Matsumoto, T. Fujii, K. Kageyama, H. Takagi, T. Nagashima, M. Hangyo, Measurement of the soft-mode dispersion in $\mathrm{SrTiO}_{3}$ by terahertz time-domain spectroscopic ellipsometry, Jpn. J. Appl. Phys. 48, 09KC11 (2009)

73. F.M.C. Gervais, B. Piriou, Temperature dependence of transverse- and longitudinal-optic modes in $\mathrm{TiO}_{2}$ (rutile), Phys. Rev. B 10, 1642 (1974)

74. T. Tsurumi, J. Li, T. Hoshina, H. Kakemoto, M. Nakada, J. Akedo, Ultrawide range dielectric spectroscopy of BaTiO3based perovskite dielectrics, Appl. Phys. Lett. 91, 182905 (2007)

75. J. Petzelt, T. Ostapchuk, I. Gregora, I. Rychetsḱy, S. Hoffmann-Eifert, A.V. Pronin, Y. Yuzyuk, B.P. Gorshunov, S. Kamba, V. Bovtun, J. Pokorný, M. Savinov, V. Porokhonskyy, D. Rafaja, P. Vaňek, A. Almeida, M.R. Chaves, A.A. Volkov, M. Dressel, R. Waser, Dielectric, infrared, and Raman response of undoped $\mathrm{SrTiO}_{3}$ ceramics: evidence of polar grain boundaries, Phys. Rev. B 64, 184111 (2001)

Cite this article as: Eric Akmansoy, Simon Marcellin, Negative index and mode coupling in all-dielectric metamaterials at terahertz frequencies, EPJ Appl. Metamat. 5, 10 (2018) 